\title{
Phage Display Based Biosensing and Bioanalysis
}

\author{
Ge Wang ${ }^{1}$, Pei Liu ${ }^{1,4}$, Han Ren ${ }^{2}$, Long Yin ${ }^{3,4}$, and Aihua Liu ${ }^{1,2,3 *}$ \\ ${ }^{1}$ Institute for Biosensing, College of Chemistry and Chemical Engineering, Qingdao University, 308 \\ Ningxia Road, Qingdao 266071, China \\ ${ }^{2}$ Department of Drug Metabolism and Analysis, School of Pharmacy, Medical College, Qingdao \\ University, Qingdao 266021, China \\ ${ }^{3}$ College of Life Sciences, Qingdao University, 308 Ningxia Road, Qingdao 266071, China \\ ${ }^{4}$ Laboratory for Biosensing, Qingdao Institute of Bioenergy \& Bioprocess Technology, Chinese \\ Academy of Sciences, Qingdao 266101, China \\ E-mail address: liuah@qdu.edu.cn
}

\begin{abstract}
:
Specific probes against targets play siginificant role in biosensing and bioanalysis. The landscape phage library f8/8 displaying random octapeptides on the pVIII coat protein of the phage fd-tet, and demonstrating many excellent features including multivalency, stability, and high structural homogeneity. In this talk, we present our recent advance in selecting specific phage clones binding with different targets such as free prostate-specific antigen (fPSA), cellulase, ubiquitous pathogens from the landscape phage library. Then we developed a series approaches such as sandwich enzyme linked immunosorbent assay (ELISA) array of "phage/fPSA/anti-PSA mAb" combining with anti-PSA $m A b$ for detecting fPSA, phage microarray for the detection of cellulose, as well as rapid, selective and sensitive colorimetric biosensing of Staphylococcus aureus. Finally their applications in biomedical application is highlighted. Taken together, the landscape phage is an attractive biomolecular probe and should become efficient and cost-effective in bioanalysis and biosensing.
\end{abstract}

Key words: phage display, fusion protein, biosensing, bioanalysis, diagnostics

\section{References}

[1] G. P.Smith, Science 228,1315-1317(1985), doi:10.1126/science.4001944

[2] H. Qi, H. Lu, H.-J. Qiu, V. Petrenko, A. Liu, Journal of Molecular Biology 417,129-143(2012), doi:

10.1016/j.jmb.2012.01.038

[3] Q.Lang, F. Wang, L. Yin, M. Liu, V. Petrenko, A. Liu, Analytical Chemistry 86, 2767-2774(2014), doi:10.1021/ac404189k

[4] H. Qi, F. Wang, V. Petrenko, A. Liu, Analytical Chemistry 86, 5844-5850(2014), doi: 10.1021/ac501265y

[5] A. Liu, G. Abbineni, C. Mao, Advanced Materials 21,1001-1005(2009), doi: 10.1002/adma.200800777

[6] P. Liu, L. Han, V. Petrenko, A. Liu, Biosensors \& Bioelectonics 82,195-203(2016), doi: 10.1016/j.bios.2016.03.075

[7] L.Han, P. Liu, H.-J. Zhang, F. Li, A.Liu, Chemical Communications 53, 5216-5219(2017), doi: 10.1039/c7cc02049j 